\title{
GridFlow: an object-oriented building evacuation model combining pre-movement and movement behaviours for performance-based design
}

\author{
MARK BENSILUM AND DAVID PURSER, \\ FRS, BRE Ltd., Garston, Watford WD25 9XX, UK
}

\begin{abstract}
Methods for estimating the time required for building evacuations range from simple calculations to highly complex computer-based models. Some of these more complex models attempt to represent the pre-movement behaviour of occupants, as well as their movement behaviours and physical movement characteristics, often using detailed rule-based algorithms, or artificial intelligence techniques. The quantification of such behaviours can be obscure and the models complex to use. Other models may ignore pre-movement behaviours. GridFlow has been developed to represent individual occupants in building spaces on a grid network, moving to exits through escape routes using an $\mathrm{x}, \mathrm{y}$ coordinate and distance map method. Walking speeds, effects of crowding, obstacle avoidance, merging flows and flows through restrictions such as corridors, stairs and doorways are represented. Physical movement methods are therefore similar to those used in other models such as Crisp, Exodus, Simulex and others. GridFlow differs from other models in that premovement time and pre-movement-travel interactions are considered central to evacuation time modelling. In GridFlow, the pre-movement time is handled in the form of distributions obtained by direct measurements during monitored evacuations. The model therefore encompasses crucial elements of the evacuation process, without requiring detailed behavioural simulations. This is considered to be as informative as the most sophisticated models, using simple, transparent and easily verifiable behavioural inputs, derived from empirical data or specified and justified by the user.
\end{abstract}

KEY WORDS: escape, evacuation, pre-movement, model, travel,

\section{INTRODUCTION}

The application of performance-based (FSE) building design has led to the development of a number of evacuation simulation models. These can be used to reveal potential problems in the physical layout and enable analysis of the sensitivity of the estimated evacuation time to various management strategies or occupant scenarios. Models available range from simple equation-based models, dealing with the population as a homogenous mass, to sophisticated simulation models, in which the building and its occupants (and sometimes a developing fire) are represented in some detail. The levels of representation of the various components (particularly the building spaces and people) vary considerably. The assumptions that must be made about these components depend to a large extent on the sophistication of their representation. Where they are represented at a high level of detail, each of the specific parameters modelled must be explicitly defined, and this in turn requires a wider assessment of the systems present in the building.

The approach used in British and ISO standards for fire engineering [1,2] is that once a building evacuation has started, evacuation time can be expressed in terms of two main components: pre-movement time (the time required for occupants to engage in a range of 
behaviours before moving to exits and escape routes) and travel time (the time required for movement to exits and through escape routes). Pre-movement includes a wide range of complex behaviours, often described qualitatively but seldom adequately quantified. Travel involves principally the physical movement of occupants, so that a basic feature of any evacuation model is the extent to which it is able to represent aspects such as walking speed, effects of crowding, obstacle avoidance, merging flows and flows through restrictions such as corridors, stairs and doorways. Behaviours such as exit choice are also important in travel calculations as well as interactions between pre-movement and travel for individual occupants. The approach to physical movement modelling employed by most evacuation models currently in use is to represent individual occupants in building spaces using a fine grid network [3-5]. Various methods are used to guide occupants to exits, including the x,y coordinate and distance map method developed for Simulex [5]. Exit choice is handled in a variety of ways, such as optimisation, random, weighting or user specification [3-5]. Most models handle building evacuation but Exodus and Crisp are probably most advanced in handling interactions between occupants and fire conditions. A limitation of most models is the extent to which premovement behaviours are handled, so that applications of evacuation models for large buildings tend to concentrate on the travel phase. Pre-movement is often ignored or handled as a simple constant.

Some models, in particular the Exit89 network model [6] and Crisp [3], were used initially to model pre-movement behaviours in considerable detail, mainly for domestic situations involving a few occupants. In such situations, the detailed behaviours of individuals may considerably affect the outcome. In large buildings, containing many occupants, population behaviours are more predictable following general alarms, so that considerably simplified behavioural aspects are often used in the models. The result is that a number of models, while in theory capable of rendering considerable behavioural and movement complexity, only make use of a few behavioural features and yet may be rather difficult to operate. Also, the quantitative and cognitive basis of the behavioural features may be somewhat obscure.

At BRE, experimental measurements of pre-movement behaviour, pre-movement time distributions and evacuation times have been made in a range of occupancies [7]. A computer evacuation simulation model "GridFlow" has been developed using a number of features of the evacuation components of the more comprehensive "Crisp" risk assessment model [3]. The design rationale, simulation methods and validation procedures for GridFlow are outlined, and some applications are introduced. GridFlow was originally developed for research on human behaviour and means of escape. The objective was to create a model able to represent the parameters considered to be most important in calculating evacuation time in sufficient detail, while remaining transparent and simple to set up and run. The increasing sophistication of the model now enables application to design cases. The essential requirements of GridFlow were that the model should represent individual occupants in building spaces on a grid network, with the ability to move occupants to exits and through escape routes using an $\mathrm{x}, \mathrm{y}$ coordinate and distance map method. The model should include aspects such as walking speed, effects of crowding, obstacle avoidance, merging flows and flows through restrictions such as corridors, stairs and doorways. To this extent GridFlow has been developed with these basic physical movement methods in ways similar to those used in other models such as Crisp [3], Exodus [4] Simulex [5] and others. The algorithms for movement and flow have been based upon empirical data and methods, particularly as described by Nelson and MacLennan in the SFPE Handbook [8].

The development of GridFlow has differed somewhat from other models in the attention given to pre-movement behaviours. Pre-movement time and pre-movement-travel 
interactions are considered central to evacuation time modelling. In GridFlow, this is handled in the form of distributions, from which different individual pre-movement times are sampled for the occupant population. The pre-movement time distributions used have been obtained by direct measurements during monitored evacuations or incident investigations [7]. The specific distributions are defined by the user or defaults may be used. By this means, GridFlow provides a simple and transparent but realistic method for rendering both pre-movement and travel aspects of building evacuations, which can easily be modified to accommodate experimental evacuation data, and provide a simple method for evaluating evacuation times. It is considered that the additional complexity of the most advanced models may not yield significantly different results. The crucial aspect is that the underlying assumptions are sound, which applies to both the advanced models and the simpler approaches.

\section{MODEL OVERVIEW}

GridFlow is an object-oriented evacuation model, written in Visual Basic, which can run on any PC. There is a simple graphical user interface (GUI), allowing the user to simply specify a building design and occupant scenario, exporting the results directly to an Excel spreadsheet. The movement algorithms employed are based on Nelson and MacLennan [8], who described a detailed method of estimating occupant movement and flows through various types of building spaces and elements.

\section{Building representation}

The building is represented as any number of individual spaces, which may be interconnected. Each space is represented as a "fine network" of 2D rectangular cells, with each cell being $0.5 \mathrm{~m} \times 0.5 \mathrm{~m}$ in size. The layout of the space is mapped onto this $2 \mathrm{D}$ grid, so that each cell can represent free floor-space, a wall or other obstruction, or an exit from the space, which may lead to another space or to a place of safety. A simple GUI allows the user to define the boundary and internal layout of each space as a series of lines representing the walls or obstructions, or exits. Each space appears in a separate window, and the view on each space can be zoomed and panned individually at any time. Copy and paste facilities are also provided.

\section{Distance map generation}

To guide occupants to their desired destination, a series of maps is generated providing directional information from every cell to all predefined destination points, taking account of any intervening obstacles. Once these maps have been generated for the entire building, each occupant is guided through the spaces, around the obstacles, to their chosen destination, which may change as the simulation progresses. Each map comprises an array of values, one for each cell, which represents the distance, in cells, from that cell to the respective destination. Walls or obstructions are represented by special values, and exits by a value of just over zero. From any given cell, therefore, the movement bearing to move in to reach the destination can be calculated from the surrounding cells. The maps are generated using a series of recursive algorithms that determine the direct distance to the destination from any point, working around any obstacles that are present.

Figure 1 shows an example distance map for a simple multi-compartment building, consisting of a two-storey office building, with open-plan and cellular offices and a staircase. Depending on the complexity of the space, and the specific nature of the investigation, the user can represent the building as a few large spaces, or as a larger number of discrete spaces linked by doorways. For example, the space shown in Figure 1 could be subdivided so that the corridors and cellular offices on each floor are represented as independent spaces. This latter approach gives a greater level of control 
over the specific flows between the spaces, and more detailed outputs on the progress of the evacuation, but increases the setting up and running times. times.

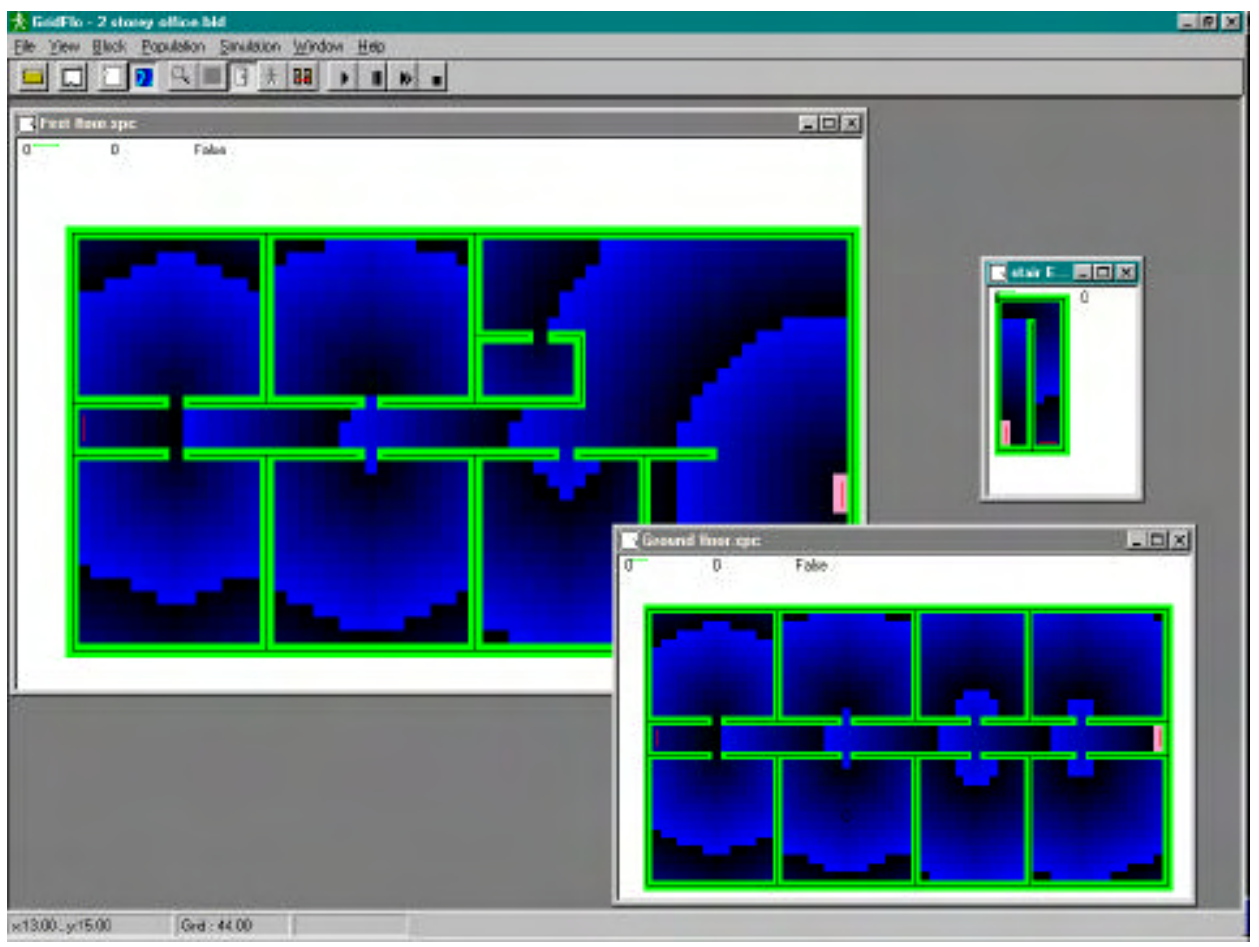

Figure 1. View of multi-compartment building, showing distance maps

\section{Exits and links}

The user can specify links from each space, which may lead to the outside (or some other place of safety), or to a link in another space. Each link has a series of properties that can be specified, including physical width and maximum throughput (in persons/second). The maximum throughput is assigned by default based on Nelson \& MacLennan's effective width method [8], but the user can override this and assign any desired value.

\section{Occupant representation}

Each occupant is represented as an individual object within the model, which has a number of associated properties. Every person is identified with a unique ID code and associated parameters including present location (space and $\mathrm{x}, \mathrm{y}$ co-ordinates), starting location, destination within current space, pre-movement time, and unimpeded walking speed. Some of these are described in more detail below.

\section{Desired destination}

The destination point to which the individual wishes to travel may be defined in various ways. The default is to move towards the nearest exit in the space, but specific or random choices can also be specified by the user. This allows particular exits to be given preference. For example the main entrance may be the only way out that is familiar to many occupants in some building scenarios. 


\section{Pre-movement time}

Pre-movement time (consisting of recognition time and response time) can be shown to be the longest component in the evacuation process in many cases [7]. Recognition time is that between an individual receiving a cue and when they cease what they were doing and begin to respond. During this phase occupants continue to do what they were doing at the time they received the cue. Response time is that between the start of reacting to the cue, and when an occupant begins to move towards an exit in order to escape. During this phase occupants may engage in a variety of activities, such as investigation, gathering friends or family, closing tills or shutting down equipment and so on. During the premovement phase occupants may be static, or they may move around looking for other people, equipment etc., or may be searching for a suitable exit. Such movement is not modelled by default in the current version of the model, although intermediate destinations can be defined prior to exiting. The outcome from this simplified approach can be equivalent to that from a more sophisticated one (which might involve complex behavioural rules or artificial intelligence) provided that the underlying assumptions used in setting the pre-movement parameters for the expected occupancy are sound.

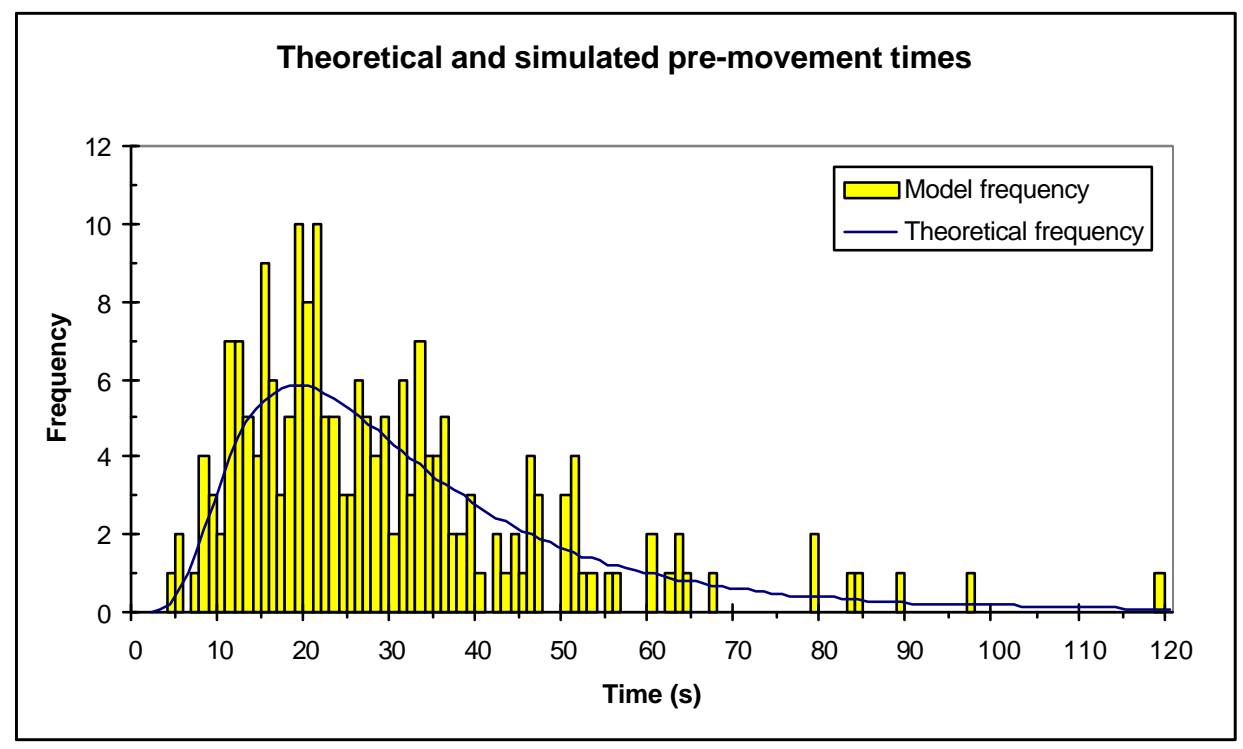

\section{Figure 2. Theoretical and modelled pre-movement distributions}

Pre-movement time can be specified as an explicit value (e.g. two minutes), or as a distribution with certain characteristics. Typically, a unimodal, positively skewed distribution would be suitable for most cases. Normal, log-normal and Weibull distributions can be supported. For example, a group can be defined which has a lognormal distribution such as that in Figure 2, which shows the theoretical frequency distribution and example of an actual sample occupancy (numbering 200 people) generated by the model. The theoretical distribution used was derived from a monitored evacuation in a department store, in which the alarm was a simple sounder, but the level of staff training was quite high, resulting in an efficient evacuation. In this example, $95 \%$ of the population had ended their pre-movement phase 74 seconds (1.24 minutes) after the start of the alarm. Clearly, on any given individual simulation, the actual frequencies generated will differ from the hypothetical ideal. Therefore it is usually necessary to 
perform several runs, generating a new sample occupancy each time, to give a more representative result. A range of user-defined groups with different characteristics can be independently created and added to the building. For example, groups representing staff and customers, or lecturers and students can be defined.

\section{Travel speed}

Each occupant is assigned an unimpeded walking speed - the speed at which they will travel across open floor-space when there are no other occupants causing an obstruction. This can be defined as a single number, or as a distribution (such as normal or log-normal positively skewed). A wide range of observed travel speeds have been reported [4,5], depending on factors such as individual differences, sex and environmental conditions. Default walking speeds are assigned from a theoretical normal distribution (mean of 1.19 $\mathrm{m} / \mathrm{s}$ and a standard deviation of $0.3 \mathrm{~m} / \mathrm{s}$, subject to a minimum of $0.3 \mathrm{~m} / \mathrm{s}$ ). This mean derived from Nelson \& MacLennan [8], is slightly conservative. Also, no standard deviation was specified. Specific or default speed factors for stairs can be assigned, reflecting the slower travel speed through these elements. User-defined groups can easily be created to give tighter control over the occupancy profile. For example, independent male and female groups can be defined, or mobility-impaired persons can be added manually as groups or as individuals. When the local occupant density increases, causing individuals to come into close proximity to one another, the movement speed is reduced.

\section{Populating the building}

Any number of independent occupant groups can be defined by the user. Each individually-named group can be assigned a range of characteristics, including the primary parameters described above, and given its own display properties to distinguish it from other occupants. For example, separate staff and visitor groups can be defined, which may have different pre-movement times, movement speeds and exit choices. Group parameters can be assigned as distributions or as discrete values. Individual occupants can also be added manually, to represent persons with specific characteristics. Once a population has been defined, single or batch simulations can be performed. Where a group characteristic has been defined in terms of a distribution, new values, drawn from the same distribution, can be assigned on each run. In this way, the effect of random variation in sample occupancies can be investigated.

\section{The simulation}

When a building has been defined, and the occupant characteristics specified, the simulation can begin. These can be carried out as single runs, or as batches of predefined scenarios. The user can choose to animate the simulation, and can select which windows to display and at what zoom level. The simulation can be paused at any time, allowing closer inspection of certain areas if desired. A range of output data can be exported directly to an Excel spreadsheet for further analysis.

\section{Movement of people}

At each $0 \cdot 1 \mathrm{~s}$ time step, all occupants whose pre-movement period has expired are moved towards their desired exit. The precise $\mathrm{x}, \mathrm{y}$ coordinates of each occupant are updated at every step. For unimpeded occupants, the distance moved will depend upon their unimpeded walking speed, in a straight line. Those in close proximity to a wall or another occupant have their speed reduced. Obstructed occupants adjust their course in order to try and avoid the hindrance. This deviation from the unimpeded course is maintained, in steadily decreasing magnitude, over a specified number of subsequent time steps. In this way, fast-moving occupants can overtake slower ones, and individuals who find themselves at the back of a queue will attempt to move forwards towards their destination 
if possible. The distance at which individuals interact is around $0.5 \mathrm{~m}$, because each person is only aware of those in the neighbouring cells. This approach reduces the processor overhead significantly. According to research by Thompson \& Marchant [5], the typical distance threshold at which movement speed starts to be affected is around $1.6 \mathrm{~m}$. Clearly, the individuals in GridFlow operate at a more localised level. However, the characteristics of the movement algorithms, which mimic the momentum of an impeded person, result in very a very similar outcome over a period of several timesteps (see section on validation).

\section{Flow though exits and other elements}

A critical parameter in any evacuation simulation is the maximum sustainable throughput of a given element, such as a door or stairway. The physical aspects of crowd movement through buildings have been relatively well described, and are fairly amenable to some kind of prediction. However, there remains a wide variance in the quantitative values used in calculating the maximum flow for a particular element. Thompson \& Marchant [5] summarised the various maximum flow rates that have been reported. In GridFlow, the flow calculation method of Nelson \& MacLennan [8] has been adopted. The flow through an exit is governed by an algorithm that prevents individuals who want to pass through from doing so until the capacity of the given element permits. For exits to the outside, no impedance beyond the doorway is assumed. Where the arrival rate at the exit is less than the maximum capacity, individuals are unimpeded. When arrival rate exceeds capacity, the flow rate is capped and a queue forms. For exits leading to another space, occupant density at the location of the inlet element is also considered. Occupants are allowed to pass through the exit only if the doorway capacity allows, and if there is sufficient space at the inlet side of the link.

Links can be used for exits from one space to another and also to govern the rate of flow at any point in a building where this may be important. For example, two links can be placed adjacent to one another in the same space, to restrict the maximum flow rate precisely to a predetermined value. This may be useful in simulating turnstiles or ticket barriers. In other parts of a space where flow may be restricted by obstacles, but which are not controlled by using links, flow rate depends upon the movement algorithms, which dictate how people move around obstructions and other individuals. These algorithms have been tuned to give flow rates that are consistent with Nelson \& MacLennan's calculation method under most circumstances. This is described in the section on validation.

\section{Merging flows}

Although the physical aspects of crowd movement through buildings are relatively well described (albeit with a fairly wide range of quantitative parameters for any given aspect), the prediction of flow rates at points where several streams of people merge is less well understood. This is a crucial factor in the evacuation of any multi-compartment building, but there is little guidance in the literature. An important case involves simultaneous evacuation from two or more floors in a multi-storey building. As the population of each floor flows into the staircases and the staircases start to fill up, the aggregate inlet flow will be restricted to the maximum sustainable outlet capacity. The division of this flow between the competing inlets is the key factor, and one for which there is little clear guidance.

Nelson \& MacLennen [8], in their worked example of a nine storey office building, suggested that, in the first instance, an assumption is made that flows on the stair dominate. This impedes entry to the stair from lower floors so that, after the staircases have filled to capacity, the flow from the highest occupied floor takes precedence, and the 
building clears from the top down. Thus, while different assumptions about merging of flows will not usually affect the predicted overall clearance time for the whole building, progress of the evacuation in specific zones within the building, may be profoundly affected. Purser \& Utting [9] highlighted the possible effects of merging at storey exits in multi-storey buildings, showing that certain floors could be seriously delayed and that the location of the delay depended upon the assumed merging ratio.

In GridFlow, there are a number of options for specifying how the merging of streams of people will be simulated. In the "free-flow" option, flows are determined by the personal movement algorithms alone, with no other special rules imposed. Where several inlets are in competition, precedence depends upon the physical arrangement of the routes, the relative widths of the links and the crowd densities at the inlets and outlets. Alternatively a "controlled" flow option can be set, which imposes additional rules on the relative dominance of competing inlets. With this option set, a staircase with two inlets (one from the staircase above and one from its storey exit) would balance the two inlets, when it was at or near its maximum occupancy. If the rate of arrival at the two inlets is sufficient, the rate of entry from each inlet would be exactly half of the outlet flow from the element. There is also provision for assigning links with a particular weighting manually, so that the relative dominance at a point of merging can be controlled. Some examples of these effects are examined in the section on validation.

\section{Outputs from the model}

A range of output data can be exported directly from the model into an Excel spreadsheet. These include a detailed break-down of the population in each space at every logging interval after each simulation run, and summarised data from a series of predefined batch runs (detailing the main features of the population and the progress of each simulation run). Also, for validation and reporting purposes, detailed data on aspects of the building (e.g. total occupiable area, capacities of inlets, travel distance distribution) and the occupants (e.g. distributions of pre-movement time, unimpeded walking speed, exit time) can be viewed or exported.

\section{VALIDATION OF THE MODEL}

All models require verification and validation, to demonstrate that they are fit for the intended task. All but the very simplest of models tend to become "black boxes", for practical or commercial reasons, so that the underlying workings of the models are not open to scrutiny. It is therefore necessary to demonstrate that the outputs from the model are consistent and reasonable. Galea et al [4] defined validation as "the systematic comparison of model predictions with reliable information", and outlined four elements of model validation (p711):

\section{Component testing}

The routine checking of major software components

2. Functional validation

Checking model capabilities and that inherent capabilities are compatible with intended use

3. Qualitative verification

Comparing predicted human behaviour with informed expectations, and

4. Quantitative verification

Detailed comparison of model predictions with reliable experimental data

Validation and verification tests on GridFlow include the following. During development of the model we have carried out many runs using simple building elements, building 
enclosures and multi-enclosure spaces which have included aspects of all four elements. Component testing and quantitative verification has included simulations of simple and more complex cases testable against empirical data as cited in the SFPE Handbook [8] and other sources. The compatibility of the model capabilities with the intended use has also been checked, and the limitations stated. For example occupants cannot modify their behaviour during the travel phase other than by collision and obstacle avoidance. Behaviour has been validated by using actual pre-movement time data and by comparison of modelled evacuation behaviour and evacuation times with observed evacuations and with Handbook data. One method of validation is to set up and populate an actual building in the model and to compare the results with those from an experimental evacuation. As Galea has stated [2,4], this has a limited application, because any specific experimental evacuation represents only one example of a distribution of outcomes that might occur on different occasions. Nevertheless, it is important to demonstrate that modelled outcomes are compatible with the main features (and times) of experimental evacuations. Some examples covering these issues are presented in the following sections.

Figure 2 shows an example of a test on a model component, in which a theoretical premovement time distribution is depicted, together with one occupant sample derived using the curve's parameters. With repetition of this process, the 'observed' frequency curve tends towards the theoretical curve. This demonstrates that the algorithm used to generate an individual random datum, drawn from a predefined distribution is functioning correctly. Similar tests have also been carried out on the other available distributions, such as the distribution of unimpeded and impeded walking speeds for an occupant sample. Other checks have been carried out on the key elements of the model, including movement across open space, through restrictions and via doorways, such that these are consistent with expectations. In the following section, some simple applications of the GridFlow model are described and used to further illustrate higher level aspects of validation including all aspects of evacuation from a single building enclosure. Similar studies have been carried out for multiple enclosures, including horizontal and vertical evacuations involving merging flows and stairs. The effects of movement features such as opposing flows and negotiation of obstacles have also been examined.

\section{Sample applications and validation for a building case}

A series of simulations has been performed to examine the interactive effects of premovement time, travel time and exit flow capacity, for different types of occupancy, using simple enclosure layouts. Figure 3 shows evacuation time outputs from three simulations for a single storey department store. The hypothetical building used here was a simple, square space $42.4 \mathrm{~m} \times 42.4 \mathrm{~m}$, giving a total area of around $1800 \mathrm{~m}^{2}$, with four exits of $1.125 \mathrm{~m}$ (one in each corner). The building features and design occupant population (900 people) (and discounting the larger main entrance) are in accordance with the rules in the UK prescriptive guidance (Approved Document B [10]). Three occupancy levels are depicted: 900,400 and 150 people. In each simulation, a sample population was generated, with a pre-movement distribution equivalent to that shown in Figure 2, and the default walking speed distribution described. Three curves are shown for each case: a pre-movement line (the cumulative number of people ceasing their premovement phase and therefore beginning travel towards an exit), a presentation line (The cumulative "presentation time" distribution) and a cumulative distribution of actual exit times. "Presentation time" represents the time at which an individual would reach an exit if their movement towards and through the exit was unimpeded by other occupants. For an individual, it is the sum of their pre-movement time and the time taken to walk to the exit. 
For a population of 150 people, the presentation and actual exit time curves are essentially coincident. This indicates that the occupants experienced little or no impedance in their progress towards the exits, and that there was no sustained queuing at the exits. In this case, therefore, the evacuation time was driven by the pre-movement and travel distributions, and not by the exit flow capacity. The rate of arrival of people at the exits is determined by the distributions of pre-movement time, distance from exits and walking speed, and for the lowest population case this rate was always lower than the maximum flow rate that the door could sustain. For a building designed within the prescriptive guidelines, the average travel distance to an exit should be short (assuming that exit choice is efficient), so the presentation time is likely to be driven mainly by the pre-movement time distribution of the occupants.

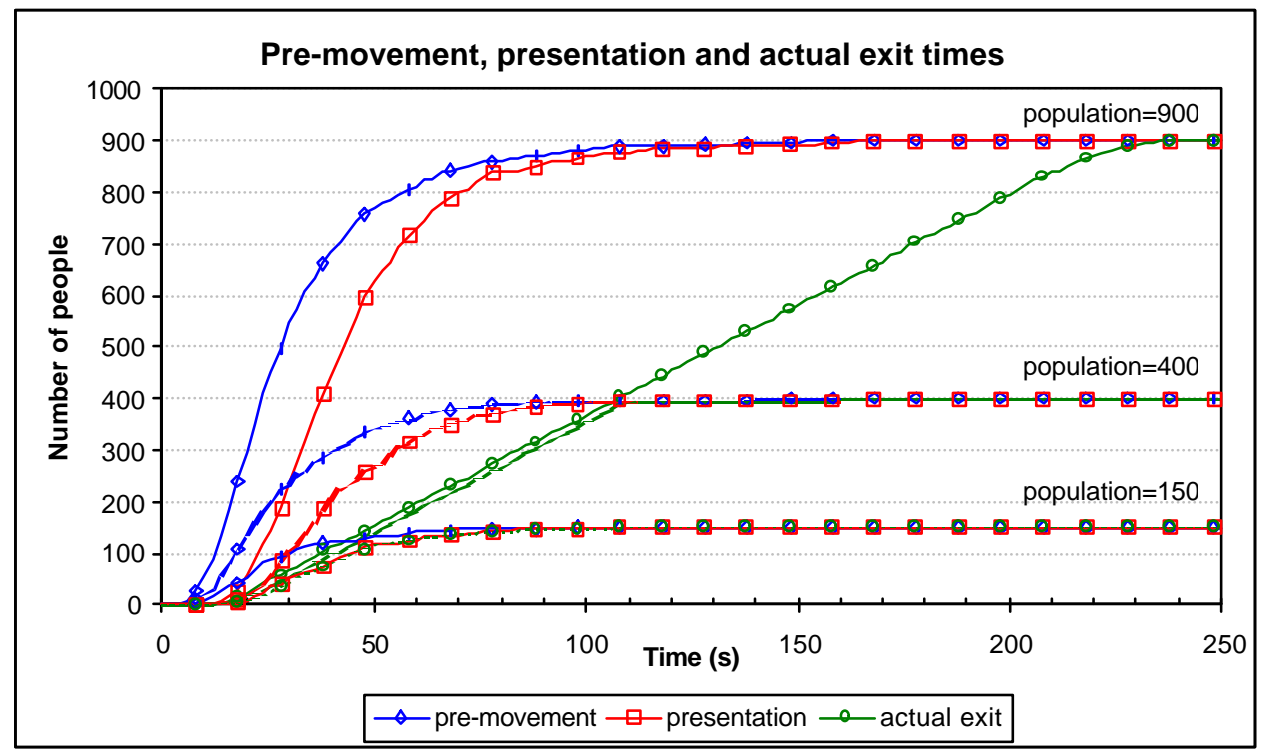

Figure 3. Effect of population on pre-movement, presentation and actual exit times

Where the population density is highest (900 people), the presentation and actual exit curves have diverged. There was a sustained period of queuing at the exits, indicated by the straight-line portion of the actual exit time curve. The gradient of this straight line has been used to validate the exit flow algorithms within the model. In this case, the exit flow capacity is driving the evacuation time. Most of the population have ceased their premovement phase well before the predicted clearance time, and the last few people to start making their way out will simply join the back of a queue. The total evacuation time can be estimated by calculating the time of queue formation (derived from the pre-movement, travel distance and walking speed distributions), and the time required for the population to pass through the exits used. For the intermediate case (population 400), the primary determinant of evacuation time is also the exit flow capacity, but the last few people to finish their pre-movement phase and travel to an exit arrived just as the queue subsided.

For any distribution of this type, the pre-movement time of the last few people to move, (i.e. those at the tail of the distribution), is inherently unpredictable. For this reason, we advocate the use of a reasonable, justifiable parameter for these "stragglers", such as the time at which $95 \%$ or $99 \%$ of the occupants would be expected to cease their premovement phase, which can be determined accurately from the underlying distribution. 
When a range of population densities are simulated, the relative importance of presentation time and flow time through the exits, for the space under consideration, can be examined. Figure 4 shows the averaged results from a series of simulations. The lower of the two horizontal dashed lines shows the time at which $95 \%$ of the occupants are expected to have ceased their pre-movement phase, and the upper the equivalent $99 \%$ time. These are determined by the selected pre-movement distribution. The continuous $\mathrm{N}$ $\& \mathrm{M}$ line shows the predicted flow time calculated using the Nelson \& MacLennan method. This only accounts for optimised flow through the exits, ignoring pre-movement and travel times (i.e.assumes zero pre-movement time and that all exits are running at maximum flow capacity throughout). The other lines show the times at which $95 \%$ and $99 \%$ of the population has exited the space respectively), and the time at which the last person exited (using a full evacuation simulation including all pre-movement, walking and exit flow variables). For low occupant densities evacuation time is dominated by presentation time.

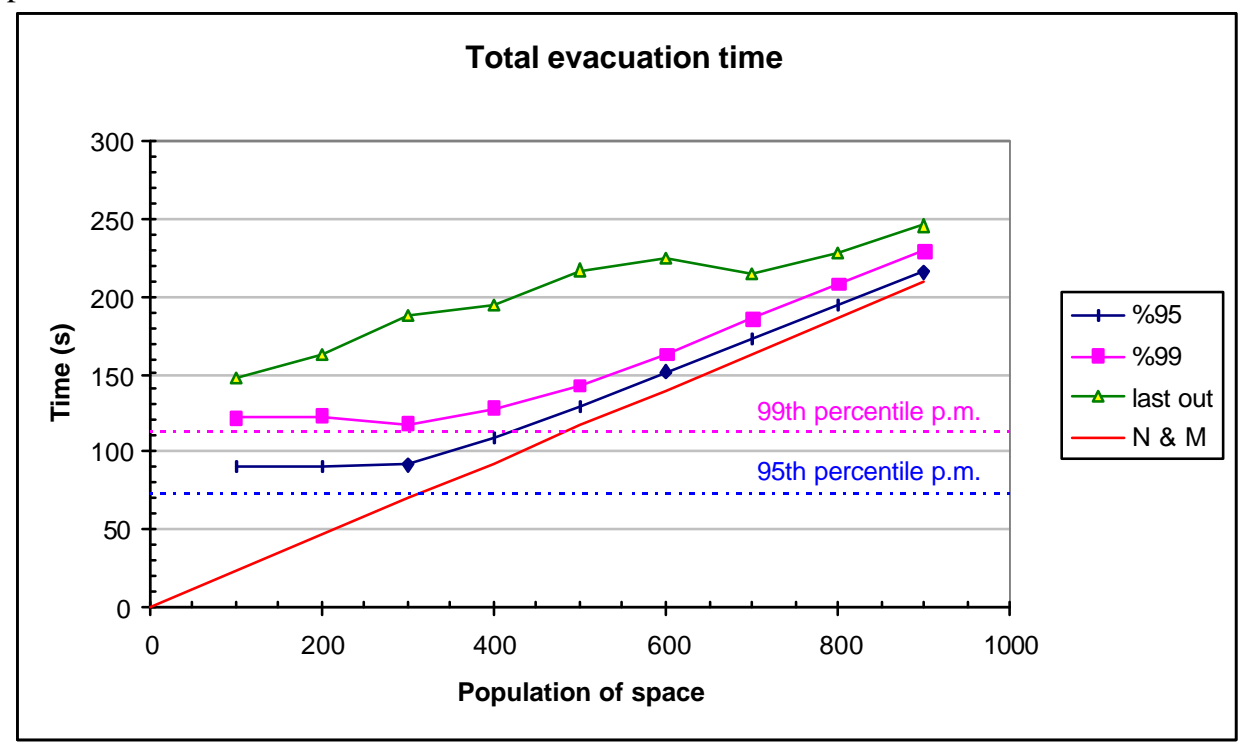

Figure 4. Total evacuation time for department store

For high occupant densities, it is dominated by the time taken for the queues to form and the exit flow time. When internal obstacles were added to the space, travel times increased for some occupants, slightly extending time to queue formation.

This example also provides an opportunity to partially validate GridFlow against experimental evacuation data. The log-normal pre-movement time distribution used in the example was derived from the observed pre-movement time distribution in a sample of occupants during the "Sprucefield" evacuation. Part of this experiment included a prescriptively designed food hall with a population of 190 occupants [7]. The worked example is not a direct simulation of the Sprucefield building, but it is sufficiently similar that the evacuation would be expected to give a somewhat similar result to the experiment. In practice the time to evacuate $99 \%$ of Sprucefield occupants was 140 seconds. This compares with the example case with a prediction of approximately 130 seconds for 190 occupants. This suggests that GridFlow is providing reasonable results, although we do plan to carry out direct simulations of the Sprucefield and other experimental cases. 
An approach such as that introduced above can begin to offer designers or assessors a simplified means for determining the driving factors for a specific scenario. Clearly the assumptions made in arriving at the occupant parameters used are crucial, and need to be carefully justified, but this is true of any model. This simplfied approach places that control fully into the hands of the user in a transparent way.

\section{CONCLUSIONS}

The aim in developing GridFlow has been to produce a model incorporating all the crucial aspects of building evacuations in sufficient detail, while still remaining transparent and easy to use. This contrasts with models containing highly complex submodels on certain aspects of crowd movement or human behaviour. It may not be necessary to include such levels of sophistication in order to get valid results, except in particular circumstances. Indeed, the scarcity of reliable data probably precludes the robust prediction of behaviour. The results from simpler methods can be as valid as those from any other but the onus is still on the user to ensure that the assumptions in the overall approach are sound.

\section{REFERENCES}

[1] "Application of fire safety engineering principles to the design of buildings" BS 7974 (and associated published document PD6 in press). British Standards Institution 2000.

[2] Fire Safety Engineering - Part 8: "Life Safety - occupant behaviour, location and condition". ISO/TR 13387-8 (1999), ISO, Geneva.

[3] Fraser-Mitchell, J.N. "Lessons Learnt During the Development of CRISP2, a MonteCarlo Simulation for Fire Risk Assessment." INTERFLAM '96 - Proceeding of the $7^{\text {th }}$ International Fire Conference. C. Franks (ed). Interscience Communications Ltd., London, UK, 1996, pp. 631-639.

[4] Galea, E.R., Gwynne, S., Owen, M., Lawrence, P.J. and Filipidis, L. "A comparison of predictions from the buildingEXODUS evacuation model with experimental data." Proceedings of the First International Symposium on Human Behaviour in Fire, 1998, pp 711-720.

[5] Thompson, P.A. and Marchant, E.W. "A computer model for the evacuation of large building populations". Fire Safety Journal, 24: 131-148 (1995).

[6] Fahy, R.F. "EXIT89 - An Evacuation Model for High Rise Buildings," Proceeding of the Third International Symposium, G. Cox and B. Langford (eds), International Association for Fire Safety Science, 1991, pp. 815-823.

[7] Purser, D.A. and Bensilum, M. "Quantification of behaviour for engineering design standards and escape time calculations", Safety Science 38:157-182 (2001).

[8] Nelson, H.E. 'Bud' and MacLennan, H.A. "Emergency movement," The SFPE Handbook of Fire Protection Engineering $\left(2^{\text {nd }} e d\right)$, DiNenno P.J. (ed) National Fire Protection Association, Quincy, MA 02269, 1995,p. 3/286-295.

[9] Purser, D.A. and Utting, S. "Required stair widths for escape routes". Building Research Establishment Report CR65/96, Garston, UK, 1996, 77 p.

[10] "The Building Regulations 2000 - Approved Document B". Department of the Environment, Transport and the Regions. London, 2000 Edition. 